\title{
Global Epidemiology of Tuberculosis: Past, Present and Future
}

\author{
Eyasu Ejeta ${ }^{1,2^{\star}}$, Mengistu Legesse ${ }^{1}$, Gobana Ameni ${ }^{1}$ and Raghavendra $\mathrm{HL}^{2}$ \\ ${ }^{1}$ Aklilu Lemma Institute of Pathobiology, Addis Ababa University, Addis Ababa, Ethiopia \\ ${ }^{2}$ College of Health and of Medical Sciences, Wollega University, Post Box No: 395, Nekemte, Ethiopia
}

\begin{abstract}
Tuberculosis (TB), one of the most widespread infectious diseases, is the leading cause of death in the world and has been known in humans since ancient time. The epidemiological trends of tuberculosis since the pre-chemotherapy era to the current both in developed countries and developing countries are presented in this review. A declining trend of magnitude of TB have been observed in most of the developed countries since the beginning of $20^{\text {th }}$ century with modification of life standard but after the introduction of chemotherapy, it changed in to upward trend because of HIV epidemic, socio-demographic factors and emergence of multi-drug resistance TB (MDR-TB). However, no such downward trend was observed in most of the developing countries including Ethiopia even after the introduction of chemotherapy, and those factors that have been led to increase the epidemiology of TB in developed countries further also led to worsening of the problem in developing countries. The Global incidence of TB showed a decline between the years of 2000-2006. However, it will be expected to increase further in the coming years, more significantly in the countries of Africa and South-East Asia including Ethiopia because of change in demographic factors (population growth), increase in the global incidence of untreatable drug resistance strain of mycobacterium and changes in age structure of the population and increase in HIV seroprevalence rates, especially in developing countries of South-east Asia and Sub-Saharan Africa. This calls for more intensive and sustained efforts at decreasing the reservoir of infection and reducing transmission through increasing health service facility and decreased contributing factories, run all the research activities will up-front in developing new tools and

strategies adequate to overcome the challenges in TB control
\end{abstract} Article Information Article History:

Received : 18-04-2013

Revised : 10-06-2013

Accepted : 17-06-2013

Keywords:

Tuberculosis,

Epidemiology

Developed Countries

Developing Countries

${ }^{*}$ Corresponding Author:

Eyasu Ejeta

E-mail:

\section{INTRODUCTION}

Tuberculosis (TB), one of the most widespread infectious diseases, is the leading cause of death in the world and has been known in humans since ancient time. The skeletal remains have been showed that prehistoric humans (4000 BC) had TB, and tubercular decay has been found in the spines of mummies from 3000-2400 BC (Zink et al., 2003). Phthisis is a Greek term for TB; around 460 BC, Hippocrates identified phthisis as the most widespread disease of the time. Genetic studies suggest that TB was present in South America for about 2,000 years (Konomi et al., 2002).

Although it was established that the pulmonary form of TB was associated with 'tubercles' by Dr. Richard Morton in 1689 (Trail, 1970), due to the variety of its symptoms, TB was not identified as a single disease until the 1820 s and was not named 'tuberculosis' until 1839 by Schönlein. The bacillus causing TB, Mtb, was identified and described on March 24, 1882 by Robert Koch (Ismael Kassim and Ray, 2004).

TB, or 'consumption' as it was commonly known, has caused the most widespread public concern in the 19th and early 20th centuries as an endemic disease of the urban poor such as, in England, in 1815 , one four deaths was caused by consumption and in 1918, one in six deaths in France were still caused by TB. In the 20th century, tuberculosis killed an estimated of 100 million people (Torrey and Yolken, 2005). Recently, over one-third of the world's population has been exposed to TB which approximately over two billion people worldwide carry the bacterium that causes TB, while about 15 
Eyasu Ejeta et al.,

million people suffering from an active infection at any one time (WHO, 2006).

Developing countries bear the burden of the TB epidemic. An estimated of $95 \%$ of TB cases and $98 \%$ of TB deaths occur in the developing world where people are especially vulnerable to TB because of poor living conditions and limited access to treatment. Just five countries-Bangladesh, China, India, Indonesia, and Pakistan-account for nearly half the new cases each year (WHO, 2006, and Raviglione and O'Brien, 2004).

Estimation shows that in sub-Saharan Africa alone, there are 1.3 million new cases of TB and 50,000 deaths annually (Porter and Adam, 1992). The young and most productive segments age groups of the population is seriously affected by the disease with the infection rate goes up to $50 \%$ in this group (Styb1o et al., 1996). In Ethiopia, TB is among the leading cause of mortality and morbidity (Azbite, 1991).

Although Mtb is the most common cause of human TB, unknown proportion of cases are due to Mycobacterium bovis (Mb) and other mycobacterial infection such as, Mycobacterium africanum, Mycobacterium canetti, and Mycobacterium microti (Acha and Szyfres, 1987).

Even though TB could be transmitted by ingesting infected meat and milk, mostly the disease is spread by aerosols created by coughing or sneezing. Transmission can only occur from people with active-not latent-TB. People with prolonged, frequent, or intense contact with patient are at particularly high risk of becoming infected, with an estimated infection rate of 22\% (Nicas et al., 2005, CDC, 2005, and Waddington, 2004).

The disease most commonly attacks the lungs (PTB) but can also affect the central nervous system, the lymphatic system, the circulatory system, the genitourinary system, bones, joints and even the skin (Herrmann and Lagrange, 2005).

This paper presents the past, present and futures global epidemiological trends of TB with the aims of providing information on its control and prevention strategies to mitigate the problems especially in the developing world.

\section{Epidemiology of Tuberculosis}

$\mathrm{Tb}$ is one of most widespread infectious disease, and a leading cause of death in the world .It has been known in human since ancient time, and it cause many public health problems throughout these times until its problem more aggravated by currently contributing factor such as, HIV epidemic,
Sci. Technol. Arts Res. J., April-June 2013, 2(2): 97-104

socio-demographic factors and emergence of multidrug resistance TB (MDR-TB).

In this paper the epidemiology of TB is presented in to five epidemiological periods. These are namely pre -chemotherapy era (before 1949), following introduction of chemotherapy (1949-1980), recent epidemiology (1980-2000), current epidemiology (2000-2006) and future epidemiology of TB (20062020).

\section{Epidemiology of TB in Pre-Chemotherapy Era Developed countries}

There are ample evidences that TB had been a serious problem in developed countries since the turn of $20^{\text {th }}$ century. Disease specific mortality data show that TB in England peaked around 1740 with about 900 TB deaths per lakh population per year. Most of the capitals of other western European countries attained their mortality peaks in first half of 19th century a decade's later (Grigg, 1958). TB mortality declined considerably from 200 - 400/lakh population per year at the turn of the century during the next 40 years in Czechoslovakia, Norway, Netherlands and other developed countries including USA. Mortality records in Canada showed $3 \%$ per annum decline in TB mortality prior to introduction of specific treatment (Styblo et al., 1969).

Information on TB morbidity during pre chemotherapy era available from few countries also indicated a decline in disease rates .In Denmark, the incidence of cases fell by $58 \%$ from 1921 to 1940. New cases of bacillary PTB also decreased in Norway from 1927 to 1947, more in pediatric age group (Raviglione et al., 1993). In Netherlands, $5.4 \%$ annual decline in annual risk of infection (ARI) was observed in the pre-chemotherapy era. Repeated tuberculin surveys in children showed $3.8 \%$ annual decline in the risk of infection in Vienna and $4.7 \%$ in Prague (Styblo, 1978). A similar decline was also observed in France (Styb1o, 1991). In fact, introduction of milk pasteurization in 1940s in most of the European countries leading to drastic reduction in transmission of bovine TB was also responsible for the decline in the prevalence rates of TB infection among human populace in these countries. Since chemotherapy was not-exists during this period and BCG vaccination does not affect transmission of infection, this trend in developed countries associated with improvement of life standered.

\section{Developing Countries}

Only a little information about TB situation and its trend during the first half of 20 century is available from developing countries because of lack of reliable data on mortality and morbidity of TB or 
Eyasu Ejeta et al.,

even prevalence of infection. In Alaska, TB mortality accounting for $35 \%$ of all deaths remained constant at 65 per lakh population from 1920 to 1950 (WHO, 1998). Tuberculin surveys carried out in Algeria and Tunisia showed negligible decline in risk of infection from 1938 to 1948 (Styb1o, 1991). Therefore, an inference can be drawn that in contrast to developed countries, there was no downward trend in the TB problem prior to chemotherapy era in the developing countries.

\section{Epidemiology of TB Following Introduction of Chemotherapy \\ Developed Countries}

After 1945, the fall in TB mortality rates accelerated in countries of Western Europe and North America (Styb1o, 1991). This coincided with introduction of Streptomycin in 1946, Para amino salicylic acid (PAS) in 1948 and isoniazid (INH) in 1952 (Chadha, 1997). The incidence of new cases of TB also showed similar declining trends in most of the developed countries. In Federal Republic of Germany, incidence rate of bacteriologically confirmed cases declined from 78 per lakh population in 1949 to 15 per lakh in 1979 (Styb1o, 1991). Similar decline was observed for bacteriologically unconfirmed and extra pulmonary cases. In Canada, complete coverage of the population with case finding and treatment was achieved by 1957. Since large proportion of population was already infected, notification rates were not immediately affected but later on declined at the rate of $10 \%$ per annum (Raviglione et al., 1993) and most of the new cases occurred in elderly and old persons. In Netherlands, morbidity of TB in younger people $(0-30$ years) fell by about $12 \%$ per year (Styblo, 1991).

In New Zealand (NZ), TB notification steadily declined after the Second World War and reached a nadir of 295 in 1988. In subsequent years, between 300 and 450 cases have been reported annually with a notification rate of around 10 per 100,000 (Turnbull, 2002).

In most of the developed countries like Canada, Federal Republic of Germany, France, Britain and Norway, $12-13 \%$ annual decline in risk of infection was observed after introduction of case finding and treatment programmes which is higher rates in relative to $4-5 \%$ decline per annum prior to chemotherapy (WHO, 1998). Similarly, in Netherlands, the risk of infection decreased rapidly by $15 \%$ per annum during 1951-1970 because of high sputum conversion rates achieved on treatment of bacillary cases (Styblo, 1978). Thus, TB problem declined rapidly after the advent of chemotherapy in developed countries, especially among children and young adults.
Sci. Technol. Arts Res. J., April-June 2013, 2(2): 97-104

\section{Developing Countries}

Morbidity data from developing countries are incomplete since only a third of the new smear positive cases are detected annually and thus cannot be relied upon for assessment of TB problem. Moreover, comparison with developed countries is undesirable since definition of a new case differs depending upon the resources available for diagnosing a case. The main source of information for studying the epidemiological situation of TB in developing countries is tuberculin surveys for estimation of ARI. The risk of TB infection remains high in most developing countries and on an average, it is estimated to be 50 to 100 times of that in developed countries. Most data from African countries indicate that the ARI after introduction of chemotherapy was around 1 to 2.5 percent. Though a slow decline has been observed in countries like Ethiopia, Cameroon, Burundi, Boatswana and moderate decline in Algeria, no such decline has been observed in other countries like Tanzania, Lesotho and rural Gambia. In addition, those of East Europe, a few of the Latin American countries, lower risks of infection at less than 1 percent have been recorded (WHO, 1998). However, the steep decline observed in earlier years in cities of Argentina appears to have been averted of late. On the other hand, ARI in Brazil on an average is high at 1-2 percent with wide variation between different regions of the country. ARI of 5$10 \%$ was observed in south and south East Asian countries in 1950s and continues to be high in most countries of this region despite availability of chemotherapy (WHO, 1998). This incensement was highest in Indonesia where an actual incensement of 3 to $4 \%$ was reported in some regions. Similarly, ARI rates were high in India with the 1 to 2.5 percentage (Chakraborty, 1993) and in Thailand more than $2 \%$ with no signs of any declining trends (WHO, 1998).

In the eastern Mediterranean region, ARI rates were high in Pakistan at around $2 \%$ and in Afghanistan at around $2.5 \%$. However, low recent levels of infection risks have been reported in most of the Middle East countries like Baharain, Libya, Kuwait and Syria after steep decline after introduction of chemotherapy. In the Western Pacific region, ARI remains high in Korea and Philippines at about $2 \%$ and $1 \%$ in China, though it was relatively low in Malaysia (WHO, 1998).

The above data suggests that though improvement in living standards and TB control activities led to reduction in ARI in some of the developing countries like in developed ones, ARI in most other countries remains high with no indication of any decline. Thus, the problem of TB remained high in most of the developing countries even after 
Eyasu Ejeta et al.,

the advent of chemotherapy. This was mainly because of low case-finding efficiency and poor treatment programmes.

\section{Recent Epidemiology of Tuberculosis}

Just when it was being projected that TB has been conquered in the west, research was stopped and funding was decreased; there was a dramatic change in epidemiological situation of TB. The declining trend of TB in industrialized countries and some of the middle-income countries since the beginning of the century witnessed a reversal after mid-eighties, because of the advent of HIV epidemic and other socio-demographic factors. There was further worsening of the TB problem in developing countries as well (Chadha, 1997). Besides, the worldwide incidence of HIV-attributable TB cases is estimated to increase from 315,000 (4\% of the total TB cases) in 1990 to 1.4 million (14\% of the total TB cases) by the year 2000. After these change in the trend of TB, WHO declared it as a global emergency in 1993 and stressed the need for concerted efforts to curb the spread of the epidemic (WHO, 2003).

\section{Developed Countries}

The recent epidemiological trend of TB in developed countries can be most appropriately illustrated by the TB situation in USA where, after a dramatic decline in the incidence of TB during early decay of $20^{\text {th }}$ century, there has been a $20 \%$ increase in the disease notification rates from 1985 to 1992 (Chadha, 1997). Most of the increase is accounted for those heavily populated urban areas that have greatest concentration of Asian Blacks and foreign-born immigrants from countries with high prevalence of TB. These are also the areas with highest incidence of reported AIDS cases. Overall $31 \%$ of the rise in incidence of TB in USA is attributed to HIV epidemic. Significantly, the median age of persons with TB decreased from 49 years in 1985 to 43 years in 1992 mainly due to increase in incidence among 25-44 years aged people, who are most affected by HIV epidemic. Increased transmission of infection has also resulted in higher incidence among 0-4 years aged children. The increase in cases of MDR- TB, mainly in urban and minority population due to rising number of intravenous drug users who also have high prevalence of HIV infection and homeless persons because of their erratic living situations and nonadherence to chemotherapy is an added variable responsible for the recent rising trend of $T B$. The isolates with drug resistance tended to come from younger persons indicating that drug resistance was associated with more recent infections.

In Australia, crude notification rate for all active TB declined from 12.2 per $1,00,000$ population in 1975 to $5.2 / 1,00,000$ in 1986 , after which the
Sci. Technol. Arts Res. J., April-June 2013, 2(2): 97-104

notification rate increased steadily to $7.6 / 1,00,000$ in 1995 (Heath, 1998).

In Canada, incidence has remained constant since 1986 while in Japan, there is slowing down of the declining trend, and in New Zealand, an actual increase in case notifications has been observed recently. Majority of the cases in these countries were reported among foreign born individuals (Turnbull, 2002). While no data is available about the impact of HIV epidemic on TB in Canada, adverse impact has been observed in Australia, New Zealand and Japan.

Similar trends have been observed in west European countries such as, England, Italy and Switzerland. In England, where notification rates were falling ever year, rose for the first time in 1991 by $4 \%$ than the previous year. Immigrants from Asia and East Europe were the worst affected. There was $20 \%$ increase in areas with highest number of AIDS patients. In Italy, where TB is common among HIV infected persons, and in Switzerland, a increase in $30 \%$ was observed from 1986 to 1990 years. High levels of HIV sero-prevalence have also been observed in certain urban areas of France and Spain leading to rise in reported TB cases (Raviglione et al., 1993).

In Eastern Europe, HIV problem is poorly defined and not yet considered as important factor for epidemiology of TB. However, increase in TB incidence has been observed in many countries mainly due to disturbed conditions because of economic and civil strife, resulting in shifting of priorities, breakdown of drug supply and health care system (WHO, 1994). Therefore, the recent trend of TB in developed countries was increase in its more public health important epidemic form as compared to just after chemotherapy era that have been more indicated from Table 1.

\section{Developing Countries}

While resurgence took place in developed world after years of continuous decline, it was never controlled in developing world and more recently, there has been further rise in TB cases, especially in Africa and South East Asia. At present, Africa account for more than $75 \%$ of dually infected persons with HIV and TB (WHO, 1994). HIV is the major cause of large increase in incidence of TB in a number of sub-Saharan countries. Some of these countries have experienced a 3 or 4 fold increase in TB case notification and deaths as a result of HIV associated TB. HIV seroprevalence rates of more than $40 \%$ are common among patients with TB in many African countries and even higher rates have been observed among extrapulmonary cases (Narain et al., 1992, and Dolin et al.,1993). Overall, 
Eyasu Ejeta et al.,

$30-40 \%$ of TB cases in Africa and Caribbean countries are attributable to HIV. In HIV affected African countries, TB has been reported in $20-44 \%$ of AIDS patients. Thus, TB is becoming one of the most common opportunistic diseases associated with AIDS (Narain et al., 1992). South East Asia and western pacific region contribute more than $60 \%$ of global TB incidence and HIV is making rapid in roads in several Asian countries including India, Thailand and Myanmar (WHO, 1994). This may result in significant increase in TB cases in Asia, as almost two-thirds of the world's TB-infected population lives in Asia. HIV seroprevalence among TB patients is on the rising in South East Asia. In one region of Thailand, it rose from $5.1 \%$ in 1989 to 14\% in 1991 (Narain et al., 1992).

High HIV seroprevalence rates have been observed in some of the Latin American countries
Sci. Technol. Arts Res. J., April-June 2013, 2(2): 97-104

like Haiti, Honduras, Argentina and Brazil (WHO, 1994). In the Eastern Mediterranean and western pacific regions, HIV seroprevalence rates among TB cases are not available for most countries.

MDR-TB, which was thought to be an insignificant problem outside USA, now spreading to different part of the world including developing countries. There are reports of rising of MDR-TB developing countries. This may further increase the problem for controlling program in these countries (Dolin et al., 1993, and Glynn et al., 2002).

The notification rate of TB for the developing countries estimated to increase in greater amount with variation between countries between 19802000 years that more indicated in below the table 2 .

Table 1: Notification rates of TB for some of developed countries between 1980 -2002 years.

\begin{tabular}{lccl}
\hline Countries & Years & $\begin{array}{l}\text { Change in rates } \\
\text { per 1,00,000 }\end{array}$ & Sources \\
\hline England and Wales & $1988-2002$ & $9.3-13.2 \%$ & Akhtar and Antoine, 2001 \\
Australia & $1986-1995$ & $5.2-7.6 \%$ & Heath et al., 1998 \\
France & $1980-2000$ & $17.2-6.12 \%$ & WHO, 2007 \\
USA & $1980-2000$ & $27.7-16.3 \%$ & WHO, 2007 \\
Italy & $1980-1998$ & $3.3-5.7 \%$ & WHO, 2007 \\
Mexico & $1980-2003$ & $1.25-18.43 \%$ & WHO, 2007 \\
Canada & $1980-2000$ & $2.76-1.67 \%$ & WHO, 2007 \\
\hline
\end{tabular}

Table 2: Notification rates of TB for some of developing countries between 1980 -2003 years.

\begin{tabular}{lccc}
\hline \multicolumn{1}{c}{ Countries } & Years & $\begin{array}{c}\text { Notification } \\
\text { rates in percent }\end{array}$ & Sources \\
\hline Ethiopia & $1980-2000$ & $40.1-91.1 \%$ & WHO, 2007 \\
South Africa & $1980-2000$ & $55.3-151.24 \%$ & WHO, 2007 \\
Kenya & $1980-2000$ & $11.05-64.16 \%$ & WHO, 2007 \\
Central Republic of Africa & $1980-1999$ & $0.66-5 \%$ & WHO, 2007 \\
Egypt & $1980-2000$ & $0.004-0.016 \%$ & WHO, 2007 \\
Brazil & $1980-2000$ & $72.6-77.9 \%$ & WHO, 2007 \\
Bangladesh & $1980-2003$ & $9.7-75.6 \%$ & WHO, 2007 \\
\hline
\end{tabular}

\section{Current Epidemiology of Tuberculosis}

According to the World Health Organization (WHO), currently nearly 2 billion people approximately one-third of the world's population have been exposed to TB pathogen. Annually, 8 million people become ill with TB, and 2 million people die from the disease worldwide (CDC, 2005). In 2004, around 14.6 million people had active TB disease with 9 million new cases while in 2005, total of 4.8 million new cases of TB were notified from all sources. This represents $55 \%$ of the 8.8 million estimated new cases; the 2.4 million new smear-positive cases notified account for $62 \%$ of the 3.9 million estimated (WHO, 2006).

The detection rate of new smear-positive cases from all sources increased slowly and linearly from 1995 to 2001 and then more quickly from 2002 to 2005 (WHO, 2007). While in 2006, 9.2 million (139 per 100 000) new cases of TB estimate occurred that including 4.1 million (62 per100 000) new smear-positive cases. Among the 9.2 million new cases of TB in 2006, WHO estimated that around 709, $000(7.7 \%)$ were HIV-positive. In terms of incident cases, India, China, Indonesia, South Africa 
Eyasu Ejeta et al.,

and Nigeria rank first to fifth (WHO, 2008). Asia (South-East Asia and Western Pacific regions) accounts for $55 \%$ of global cases, and Africa accounts for $31 \%$; the other three regions account for relatively small fractions of global cases.

In 2000, it has been estimated that approximately $40 \%$ of HIV-attributable TB cases has been occurred in sub-Saharan Africa and $40 \%$ in Southeast Asia (Dolin et al., 1993).The rest of the African Region accounted for a further $61 \%$ of HIVinfected TB cases in 2005, and $19 \%$ of all cases of TB in adult HIV-positive people was live in South Africa . Like to previous years, currently in 2006, the African Region accounts for most HIV-associated TB case $(85 \%)$. The remaining most of cases $(6 \%)$ are in the South-East Asia Region, mainly in India.

The current trend of TB was more indicted from the data of TB patient that registered in national surveillance systems and reported to WHO between 1980 and 2005, where 90 million TB patients registered, from these more than 26 million were notified by DOTS programmes since 1995 . This vast body of surveillance data suggests that the global TB incidence rate peaked some time between 2000 and 2005, although the total number of new cases is still rising each year. The global TB epidemic is now on the threshold of decline (WHO, 2008). This was more indicated from the data from 93 countries from 1997 to 2006 years which indicated that global incidence rate of TB peaked around 2002 and appears now to have stabilized or begun to decline. In 66 of these 93 countries, the rate of decline was between zero and $6 \%$ per year WHO, 2007, and WHO, 2008). Similarly, incidence rate is now stable or falling in all six WHO regions. However, the slow decline in incidence rates per capita is offset by population growth. Consequently, the number of new cases arising each year is still increasing globally and in the WHO regions of Africa, the Eastern Mediterranean and South-East Asia (WHO, 1994).

Therefore, the current decline in TB epidemiology may be promising result in the control of TB in the future, and the supposed contributing factor for this change in the trend of TB are increasing in Global DOTS and children BCG Vaccination coverage, increasing in health services centers, increasing in human awareness, and the strength of the national and Global controlling program of TB.

\section{Future Epidemiology of Tuberculosis}

Even though, the global TB incidence shows some decline from 2000-2006, it will be expected to be worsen in the immediate future, as a result of demographic factors (population growth), MDR and
Sci. Technol. Arts Res. J., April-June 2013, 2(2): 97-104

changes in age structure of the population and increase in HIV sero-prevalence rates, especially in developing countries of South-east Asia and SubSaharan Africa (WHO, 2007).

Dye models show that TB is on the increasing and that the number of cases grew $1.7 \%$ per year from 1997 to 2000 and the trends indicted that more 9-10 million new cases will be occurred in 2010 (McKenna et al., 1995). This shows that the incidence of TB will increase by $17 \%$ in 2010 in comparing to 2000 . Three fourths of this increase is expected to be due to demographic factors and the rest from a balance between decline in incidence due to intervention measures and increase in incidence due to HIV epidemic. Much higher relative increase is predicted in developing countries compared to Western Europe and other developed countries. Africa and south East Asian regions are predicted to account for three -fourths of this increase in global incidence of TB (Chadha, 1997).

In addition, as the drug resistance spreads, TB threatens to become an incurable disease for future generations and TB deaths will rise further, also because of higher mortality of HIV associated TB (Dolin et al., 1993). WHO (2005) estimates that, if unchecked, the current TB epidemic would infect 1 billion people between 2000 and 2020, of whom 200 million will develop fully active TB and 35 million will die (Joana et al., 2004).

\section{Epidemiology of Tuberculosis in Ethiopia}

According to the WHO (2008) estimate, Ethiopia ranks top $7^{\text {th }}$ in the world, and top $3^{\text {rd }}$ in Africa in the lists of high burden countries for TB, with estimated incidence of 379 per 100,000 populations for all forms TB and 162 per 100,000 populations for smear positive TB. According to this estimate, the national trend of TB incidence shows -1.3\% decreases in the years from 2005-2006. The prevalence and mortality of all forms of TB was estimated to be 643 and 84 per 100,000 populations, respectively [33]. Besides, according to National TB report, in the years 2006/7 in Ethiopia a total of 126,106 TB cases was notified to the National Tuberculosis and Leprosy Control Program (NTLCP), which was lower than the 2005/6 notification where 126,233 TB cases were notified. From the 2006/7 notified TB cases, 36\% $(45,112)$, $30 \%(377,915)$ and $34 \%$ were ETB, new smear positive PTB and new smear negative PTB, respectively (WHO, 2005).

According to the FMOH (Federal Minster of Health) hospital statistical data, TB is the leading causes of morbidity, the third causes of hospital admission (after deliveries and malaria), and the second causes of death in Ethiopia (after malaria). TB is an obstacle to soci-economic development in 
Eyasu Ejeta et al.,

Ethiopia, sine this disease affect $75 \%$ of cases with in economically productive age group of $15-54$ years (FMOH, 2007).

Another challenge of TB control in Ethiopia is the emergence of multi-drug resistant TB (MDR-TB). According to the data from the survey conducted in the country between 2003-2006, the levels of MDRTB were $1.6 \%$ and $11.8 \%$ in new and re-treatment cases of TB patients, respectively $(\mathrm{FMOH}, 2007)$.

The national standard TB prevention and control program is incorporated in to the DOTS program was started as pilot in Arise and Bale Zone, Oromia Regional State in 1992. This strategy has been subsequently scaled up in the country and currently reached at the level of $90 \%$ of geographical and $75 \%$ of health facility coverage. Besides, to strength the national TB control strategies combination of TB and leprosy control strategies was decided in 1994 as National TB and Leprosy Control Program (NTLCP) (FMOH, 2007).

\section{CONCLUSION}

TB has been present in humans since ancient history and it is becoming one of a leading life threat disease both in developed and developing countries especially in the era of HIVIADIS. It causes most widespread public concern and problem in the human history including in the first decay of 21 centuries as an endemic disease in many part of both developed and developing countries.

In the early 20century, before introduction of chemotherapy, the trend of TB shows to decline in developed countries due to change in life standard while it is still great problem in developing countries, and many activities that had been done to control TB are stopped in developed countries that lead to TB resurgence in developed counties in line with change in Scio-demographic, emergence of MDRTB, and HIV epidemic, and these factors aggravated further the problem in developing countries. Thus, TB is one of a leading health problem both in developing and developed countries throughout human history.

\section{REFERENCES}

Acha, P.N., Szyfres, B. (1987). Zoonotic tuberculosis. In Zoonoses and communicable diseases common to man and animals. 2nd edition. Washington: Pan American Health Organization/ World Health Organization. Pp 503.

Akhtar, M., Antoine, D. (2003). Preliminary report on tuberculosis cases reported in 2001 in England, Wales and Northern Ireland. Enhanced Tuberculosis Surveillance. Tuberculosis Section. CDSC London.

Azbite, M. (1991).Tuberculosis survey in Ethiopia. Akaka, 67:8539-8544.
Sci. Technol. Arts Res. J., April-June 2013, 2(2): 97-104

Centers for Disease Control (CDC). (2005). Fact Sheet: Tuberculosis in the United States.

Chadha, V.K. (1997).Global trends of tuberculosis- an epidemiological review. NTI Bulletin, 33:11-18.

Chakraborty, A.K. (1993) .Tuberculosis situation in India: Measuring it through time. Indian Journal of Tuberculosis, 40:2117.

Dolin, R.J., Raviglione, M .C., Kochi A. (1993). A review of current epidemiological data and estimation of future TB incidence and mortality; WHO/TB/93.173.

Fedral Minster of Health (FMOH). (2008). Manual of Tuberculosis, Leprosy and TB/HIV prevention and control programme. $4^{\text {th }}$ edition. p1-90.

Glynn, J.R., Whiteley, J., Bifani, P.J., Kremer, K., Van Soolingen D. (2002). Worldwide occurrence of Beijing/W strains of Mycobacterium tuberculosis: a systematic review. Emerging Infectious Diseases 8: 8.

Grigg, E.R.N. (1958). The arcana of tuberculosis with abrief epidemiological history of the disease in USA. Ame. Rev .Resp. Dis., 78: 151.

Heath, T.C., Roberts, C., Winks, M., Capon, A.G. (1998). The epidemiology of tuberculosis in New South Wales 1975-1995: the effects of immigration in a low prevalence population. International Journal of Tuberculosis and Lung Disease 2: 647-654.

Herrmann, J., Lagrange, P. (2005). Dendritic cells and Mycobacterium tuberculosis: This is the Trojan horse?". Pathologie Biologie (Paris), 53: 35-40.

Ismael Kassim., Ray, C.G. (editors) (2004). Sherris Medical Microbiology ( $4^{\text {th }}$ ed.). McGraw Hill. ISBN 08385-8529-9.

Joana, G., Thomas, N., Hiwote, T., Anatoly, V. (2004) HIVIAIDS and Tuberculosis in Central Asia Country Profiles. The World Bank working paper, Washington, D.C.

Konomi, N., Lebwohl, E., Mowbray, K., Tattersall, I., Zhang, D. (2002). Detection of mycobacterial DNA in Andean mummies. Journal of Clinical Microbiology 40: 4738-40.

McKenna, M.T., McCray, E., Onorato, I. (1995). The epidemiology of tuberculosis among foreign-born persons in the United States, 1986 to 1993. New England Journal of Medicine 332:1071-1076.

Narain, J.P., Raviglione, M.C., Kochi, A. (1992). HIVassociated tuberculosis in developing countries, epidemiology and strategies for prevention. Journal of Tuberculosis and Lung Disease 73: 311-321.

Nicas, M., Nazaroff, W.W., Hubbard, A. (2005). Toward understanding the risk of secondary airborne infection: emission of respirable pathogens. Journal of Occupational and Environmental Hygiene 2: 143-154.

Porter, J., Adam, K. (1992). Tuberculosis in Africa in the ALDS era: the role of chemotherapylaxis. Royal Society of Tropical Medicine and Hygiene, 86:466469. 


\section{Eyasu Ejeta et al.,}

Raviglione, M.C., O'Brien, R.J. (2004). "Tuberculosis", in Kasper, D.L., Braunwald, E., Fauci, A.S., Hauser, S.L., Longo, D.L., Jameson, J.L., Isselbacher, K.J. (eds). Harrison's Principles of Internal Medicine, 16th ed., McGraw-Hill Professional, 953-66.

Raviglione, M.C., Sudre, P., Reider, H.L., Spinaci, S., Kochi A. (1993) .Secular trends of tuberculosis in Western Europe. Bulletin of the World Health Organization 71: 297-306.

Styblo, K. (1991) Epidemiology of tuberculosis. Pub. by Royal Nether. TB. Association, Holland, 24.

Styblo, K., Frencly, D., Petty, T. (1996). Tuberculosis control and surveillance. In: Recent advances in the respiratory medicine. Edinburgh Churchill Livingstone 5:77-108.

Styblo, K., Meijer, J., Sutherland, I. (1969). The transmission of tubercle bacilli: Its trend in a human population: Tuberculosis Surveillance Research Unit, Report. Bull IUAT, 42: 5-104.

Styblo, K. (1978).Recent advances in epidemiological research in an urban community. Indian Journal of Tuberculosis 25: 77.

Torrey, E.F., Yolken, R.H. (2005). Their bugs are worse than their bite. Washington Post, 3, B01.

Trail, R. (1970). "Richard Morton (1637-1698)". Medical History 14: 166-74.

Turnbull, F. (2002). The Epidemiology and Surveillance of Tuberculosis in New Zealand. Guidelines for Tuberculosis Control in New Zealand 2003.
Sci. Technol. Arts Res. J., April-June 2013, 2(2): 97-104

Wellington: Ministry of Health: 1-30. URL: http://www.moh.govt.nz/moh.nsf/.

Waddington, K. (2004). "To stamp out "so terrible a malady": bovine tuberculosis and tuberculin testing in Britain, 1890-1939". Medical History 48: 29-48.

World Health Organization (WHO). (1988). Cauthan GM., Pio A., Ten Dam HG. Annual risk of tuberculosis infection; WHO/TB/88,154.

World Health Organization (WHO). (2005). Global TB Report 2004.

World Health Organization (WHO). (2006). Global tuberculosis control - surveillance, planning, financing WHO Report.

World Health Organization (WHO). (2007).Global tuberculosis control: surveillance, planning, finances. WHO report 2007, Geneva, WHO/HTM/TB/.376.

World Health Organization (WHO). (2008).Global tuberculosis control: surveillance, planning, financing: WHO report 2008. WHO/HTM/TB/2008.393.

World Health Organization. (WHO). (1994). TB - A Global Emergency. WHO Report on the Tuberculosis Epidemic. WHO/TB/945.177, Geneva, 1994.

World Health Organization. (WHO). (2003). Global TB Control. WHO/CDS/TB/.316. Geneva, 2003.

Zink, A., Sola, C., Reischl, U., Grabner, W., Rastogi, N., Wolf, H., Nerlich, A. (2003). "Characterization of Mycobacterium tuberculosis complex DNAs from Egyptian mummies by spoligotyping". Journal of Clinical Microbiology 41: 359-67. 